\title{
Central amygdala lesions inhibit pontine nuclei acoustic reactivity and retard delay eyeblink conditioning acquisition in adult rats
}

\author{
Joseph M. Pochiro ${ }^{2}$ - Derick H. Lindquist ${ }^{1,2}$
}

Published online: 20 October 2015

(C) Psychonomic Society, Inc. 2015

\begin{abstract}
In delay eyeblink conditioning (EBC) a neutral conditioned stimulus (CS; tone) is repeatedly paired with a mildly aversive unconditioned stimulus (US; periorbital electrical shock). Over training, subjects learn to produce an anticipatory eyeblink conditioned response (CR) during the CS, prior to US onset. While cerebellar synaptic plasticity is necessary for successful EBC, the amygdala is proposed to enhance eyeblink CR acquisition. In the current study, adult Long-Evans rats received bilateral sham or neurotoxic lesions of the central nucleus of the amygdala (CEA) followed by 1 or 4 EBC sessions. Fear-evoked freezing behavior, CS-mediated enhancement of the unconditioned response (UR), and eyeblink CR acquisition were all impaired in the CEA lesion rats relative to sham controls. There were also significantly fewer c-Fos immunoreactive cells in the pontine nuclei (PN) - major relays of acoustic information to the cerebellum-following the first and fourth EBC session in lesion rats. In sham rats, freezing behavior decreased from session 1 to 4 , commensurate with nucleus-specific reductions in amygdala Fos + cell counts. Results suggest delay EBC proceeds through three stages: in stage one the amygdala rapidly excites diffuse fear responses and PN acoustic reactivity, facilitating cerebellar synaptic plasticity and the development of eyeblink CRs in stage two, leading, in stage three, to a diminution or stabilization of conditioned fear responding.
\end{abstract}

Derick H. Lindquist

lindquist.40@osu.edu

1 Department of Psychology, The Ohio State University, 1835 Neil Avenue, Room 49, Columbus, OH 43210, USA

2 Department of Neuroscience, The Ohio State University, 1835 Neil Avenue, Room 49, Columbus, OH 43210, USA
Keywords Pavlovian conditioning · Eyeblink conditioning · Fear conditioning $\cdot$ Cerebellum $\cdot$ Amygdala

Different forms of associative learning and memory rely on distinct but distributed neural substrates and circuits (Fanselow \& Poulos, 2005; Rudy, 2009; Stanton, 2000). For example, learning to fear specific stimuli or events depends on the amygdala (Duvarci \& Pare, 2014; LeDoux, 2014), whereas simple forms of sensorimotor learning, such as eyeblink classical conditioning, depend on the cerebellum and brainstem (Christian \& Thompson, 2003; Freeman \& Steinmetz, 2011). In delay eyeblink conditioning (EBC), a light or tone conditioned stimulus (CS) is repeatedly paired with a mildly aversive corneal air puff or periorbital shock unconditioned stimulus (US). Over hundreds of training trials the CS comes to elicit an adaptive eyeblink conditioned response (CR), with maximal eyelid closure occurring just before the expected US.

The neural circuitry responsible for eyeblink CR acquisition and expression has been extensively described over the last half century (reviewed in Steinmetz \& Lindquist, 2009). Briefly, the CS signal is relayed to the cerebellum by pontine nuclei (PN) mossy fibers (Aitkin \& Boyd, 1978; Steinmetz \& Sengelaub, 1992) while the US signal is relayed via climbing fibers from the inferior olive (Türker \& Miles, 1986; Yeo, Hardiman, \& Glickstein, 1986). Accordingly, the CS and US signals converge on Purkinje cells in the cerebellar cortex, HVI and the anterior lobe in particular, and interpositus nucleus (IP) projection neurons (Thompson \& Steinmetz, 2009). Learning-dependent increases in IP neuronal firing activate the downstream motor nuclei responsible for eyelid closure (Berthier \& Moore, 1990; McCormick \& Thompson, 1984), while inhibitory output from the cerebellar cortex modulates the amplitude and timing of the eyeblink CR (Green \& Steinmetz, 2005; Perrett, Ruiz, \& Mauk, 1993). 
Two-stage models of aversive Pavlovian conditioning posit the rapid emergence of amygdala-dependent emotional responses in stage one, which accelerates the acquisition of more slowly acquired cerebellar-dependent sensorimotor responses in stage two (Boele, Koekkoek, \& De Zeeuw, 2010; Lennartz \& Weinberger, 1992; Mintz \& Wang-Ninio, 2001; Rescorla \& Solomon, 1967; Thompson et al., 1987; Wagner \& Brandon, 1989). In eyeblink conditioned subjects, for instance, a number of fear CRs are observed during the first training session - before significant numbers of eyeblink CRs are produced - including autonomic changes in heart rate and blood pressure, elevated freezing, and $22 \mathrm{kHz}$ ultrasonic emissions (Britton \& Astheimer, 2004; Lee \& Kim, 2004; Lindquist, Mahoney, \& Steinmetz, 2010; Prokasy, 1972; Schneiderman, 1972). As predicted by the two-stage model, amygdala damage or pharmacological inactivation diminishes fear responding and retards eyeblink CR acquisition (Blankenship, Huckfeldt, Steinmetz, \& Steinmetz, 2005; Chachich \& Powell, 1998; Lee \& Kim, 2004; Neufeld \& Mintz, 2001; Weisz, Harden, \& Xiang, 1992). No studies, to our knowledge, have reported that amygdala damage or inactivation prevents delay $\mathrm{EBC}$, signifying the amygdala contributes to (but is not necessary for) the acquisition or expression of discrete motor responses.

The precise mechanism(s) by which the amygdala facilitates cerebellar-dependent motor learning is not known, but different functions have been attributed to individual amygdala nuclei. The emotional significance (or motivational value) of the aversive US is thought to be encoded within the basolateral nucleus (BLA), which promotes association of the CS-US signals in the lateral nucleus (LA), increasing the arousal or attention directed at the CS via activation of central nucleus (CEA) projection neurons (Blair, Sotres-Bayon, Moita, \& LeDoux, 2005; Penzo, Robert, \& Li, 2014; Rorick-Kehn \& Steinmetz, 2005; Sah \& Armentia, 2003). The CEA could potentiate CS-evoked activity in the PN, the last site outside the cerebellum where CS sensory afferents converge, through monosynaptic and/or polysynaptic projections (Berger, Alger, \& Thompson, 1976; Kandler \& Herbert, 1991; Mihailoff, Kosinski, Azizi, \& Border, 1989). In Taub and Mintz (2010), for instance, tone-evoked PN unit reactivity was increased during CS-US paired but not unpaired trials, while CEA inactivation (via lidocaine) prevented the increase in PN unit reactivity during presentation of the tone CS.

In the current study, the potentiated CEA unit activity that underlies fear CR expression (e.g., freezing) was hypothesized to increase the population of PN neurons activated in response to the tone CS, promoting enhanced CS-US synaptic plasticity in the cerebellum and the emergence of eyeblink CRs. Bilateral neurotoxic CEA lesions, or sham surgery, was performed one week prior to the start of delay EBC. All rats were submitted to one or four delay $\mathrm{EBC}$ sessions and then sacrificed in order to relate each subject's behavior to posttraining neuronal activity. PN tissue was submitted to immunohistochemical (IHC) processing for c-Fos, an immediate early gene whose expression was used as an indirect marker of neuronal activity (Bullitt, 1990). If amygdala output regulates fear responding and PN acoustic reactivity, then CEA lesion rats should freeze less, have fewer Fos+ PN neurons, and demonstrate impaired EBC acquisition relative to sham controls. Furthermore, differential activation of individual amygdala nuclei across delay EBC, as suggested above, should alter the degree and persistence of neuronal activity in each nucleus. Fos+ neurons in the BLA, CEA, and LA were therefore counted and analyzed following Sessions 1 and 4 in sham rats.

\section{Materials and method}

\section{Subjects}

Experimentally naïve male Harlan Laboratories (Indianapolis, IN, USA) Long-Evans rats were individually housed and maintained in The Ohio State University Psychology vivarium. Subjects were maintained on a $12 \mathrm{hr}$ light/dark cycle (lights on at $0600 \mathrm{hr}$ ) with ad libitum access to food and water. Surgical and behavioral procedures were conducted during the light phase. All procedures, including surgery and postoperative care, were approved by and in strict compliance with OSU institutional animal care and use committee (IACUC) animal care guidelines, and all necessary measures were taken to minimize pain and discomfort.

\section{Surgical procedures}

Based on prior EBC literature utilizing amygdala lesions or inactivation (e.g., Blankenship et al., 2005; Lee \& Kim, 2004), we estimated 8 to 10 rats per group would provide sufficient power to detect significant impairments in conditioned fear and delay EBC. Approximately one week after arrival, all subjects (weighing 320-340 g) were anesthetized with respiratory isoflurane $\left(1 \%-3 \%, 0.6 \mathrm{l} / \mathrm{min}^{2}\right)$ and secured in a stereotaxic frame. The scalp was incised and two burr holes drilled in each hemisphere. Bilateral excitotoxic CEA lesions were done using the following coordinates, relative to Bregma (in $\mathrm{mm}$ ): $\mathrm{A} / \mathrm{P}:-2.1, \mathrm{M} / \mathrm{L}, \pm 4.3, \mathrm{D} / \mathrm{V},-8.2$; and $\mathrm{A} / \mathrm{P},-2.4, \mathrm{M} / \mathrm{L}$, $\pm 4.3, \mathrm{D} / \mathrm{V},-8.5$. A $1.0 \mu \mathrm{l}$ Hamilton syringe, attached to a Micro4 microsyringe pump controller (model UCM4, World Precision Instruments, Sarasota, FL, USA), was lowered into each drop site. A total volume of $0.1 \mu$ of ibotenic acid (Thermo Fisher Scientific, Geel, Belgium), dissolved in PBS vehicle $(10 \mu \mathrm{g} / \mu \mathrm{l})$, was injected in each hole at a rate of $0.1 \mu \mathrm{l} /$ min. The syringe was left in place an additional 2 min to allow for dispersal of the drug prior to its removal. Sham lesion rats received bilateral infusions of PBS vehicle at the same rate 
and volume using the same coordinates. Once injections were complete, the burr holes were sealed with bone wax.

Next, each subject was surgically prepared for EBC. Electromyogram (EMG) activity was recorded in the orbicularis oculi (OO) muscle that surrounds the left eye by passing two ultrathin (0.003-in) Teflon-coated stainless steel wires beneath the anterior portion of the upper eyelid. For delivery of the US periorbital electrical shock, gold-coated stainless steel wires were implanted in the dorso-caudal portion of the $\mathrm{OO}$ muscle. A ground wire was connected to one of three stainless steel skull screws. The two EMG wires and ground wire all terminated in gold pins inside a 6-pin plastic connector (M363; Plastics One Inc. Roanoke, VA). The headstage and bipolar stimulating electrodes were fixed in dental cement. The wound was salved with antibiotic ointment (Neosporin, Johnson \& Johnson, New Brunswick, NJ), and rats were administered an oral dose (in water bottles) of Meloxicam (1 mg/kg; The Ohio State University Veterinary Services) for two consecutive days. All subjects were given at least 7 days to recover and handled the 2 days immediately prior to the start of training.

\section{Conditioning apparatus}

Eyeblink conditioning took place in standard operant boxes (Coulburn Instruments, Allentown, PA), contained within sound-attenuating chambers. Each operant box had two stainless steel walls, two Plexiglas walls, and a grid floor composed of $0.5 \mathrm{~cm}$ stainless steel bars placed approximately $1.5 \mathrm{~cm}$ apart. The interior of each chamber was illuminated by an infrared light source, and behavior was recorded with a black-and-white video camera with a wide-field lens (Model WDSR-2005SC; Circuit Specialists, Inc., Mesa, AZ). The video signal was inputted to FreezeScan (CleverSys, Inc., Reston, VA), a software package that provides precise motion detection, quantifying the percentage of time that rats are motionless. The headstage of each rat was attached to a six channel commutator (SL6C/SB, Plastics One, Inc., Roanoke, VA, USA), allowing subjects to move freely in the conditioning chamber.

\section{Behavioral training, testing, and analyses}

Following surgery, rats were pseudo-randomly assigned to undergo one or four EBC sessions. Each chamber was sprayed with Windex prior to placing the rat inside. All rats experienced an initial 50 min stimulus-free adaptation session, followed by one session or four consecutive sessions of delay EBC, separated by $24 \mathrm{hr}$. Each EBC session consisted of 10 blocks of 12 trials: $10 \mathrm{CS}$-US paired, $1 \mathrm{CS}$-alone, and 1 USalone. The CS was a $450 \mathrm{~ms}, 2.8 \mathrm{kHz}, 80 \mathrm{~dB}$ tone and the US was a coterminating train of $100 \mathrm{~ms}, 2.0 \mathrm{~mA}, 60 \mathrm{~Hz}$, constantcurrent square wave periocular electrical stimulation. The interstimulus interval (ISI), from CS onset to US onset, was $350 \mathrm{~ms}$ and the intertrial interval (ITI) was $25 \pm 5 \mathrm{~s}$.

On each trial, eyelid EMG activity from the OO muscle was sampled for 1,500 ms, divided into three periods: (i) a $350 \mathrm{~ms}$ pre-CS period; (ii) a $350 \mathrm{~ms}$ CS-US period; and (iii) an $800 \mathrm{~ms}$ post-US period. For each trial, the averaged EMG activity in the pre-CS period was used as a baseline for classifying blink behavior. Trials were excluded from analysis if EMG activity exceeded baseline activity by 5 or more standard deviations during the bad trial window, extending from $100 \mathrm{~ms}$ before and up to $20 \mathrm{~ms}$ after CS onset. Elevation of EMG activity within the first $100 \mathrm{~ms}$ after CS onset was considered an alpha (startle) response and not counted as an eyeblink CR. A conditioned blink was scored only if EMG activity exceeded baseline activity by 5 or more standard deviations beginning $100 \mathrm{~ms}$ after CS onset through the end of the CS-US period. CR peak amplitude data was based on CSalone trials; UR peak amplitude was measured following US offset for US-alone and CS-US trials.

Freezing was defined as cessation of all visible body or vibrissae movements except that required for respiration (Fanselow, 1980). Freezing behavior was measured and analyzed in two ways: during the initial $60 \mathrm{~s}$ of each session (baseline), prior to the first CS-US trial, representing context-dependent fear behavior; and across the entire session, representing a combination of context- and CS-evoked freezing.

\section{Histology}

Subjects were sacrificed $1 \mathrm{hr}$ after the first or fourth EBC session using $200 \mathrm{mg} / \mathrm{kg}$ of Euthasol (Virbac, Fort Worth, TX, USA) and immediately perfused with $0.9 \%$ saline followed by $4 \%$ paraformaldehyde. Brains were stored in $4 \%$ paraformaldehyde and then transferred 2 days later to $4 \%$ paraformaldehyde $/ 30 \%$ sucrose for an additional 2 to 3 days. A Leica vibrating microtome (VT1000S, Buffalo Grove, IL, USA) was used to section tissue $(40 \mu \mathrm{m})$ through the amygdala and the PN. Tissue sections were stored at $-20{ }^{\circ} \mathrm{C}$ in a cryoprotective buffer containing ethylene glycol until processed for immunohistochemistry (Koo, Han, \& Kim, 2004).

To determine the placement and extent of CEA lesions, tissue sections were stained for anti-NeuN antibody (MAB377; Merck Millipore, Billerica, MA, USA). In vertebrates, NeuN is a neuron-specific nuclear protein expressed in most neuronal cell types, making it an excellent marker for identifying neurons (Mullen, Buck, \& Smith, 1992). Tissue sections from lesion rats were quenched in $0.3 \% \mathrm{H} 2 \mathrm{O} 2$ / $50 \%$ methanol and blocked in $5 \%$ normal goat serum (Vector Laboratories, Burlingame, CA, USA) for $1 \mathrm{hr}$. Sections were incubated overnight at $4{ }^{\circ} \mathrm{C}$ with $1 / 200$ dilution of anti-NeuN antibody. The next day, sections were incubated in $1 / 200$ dilution of biotinylated antimouse secondary antibody 
(BA-2001; Vector Laboratories, Inc., Burlingame, CA, USA) for $1 \mathrm{hr}$ at room temperature and then rinsed three times in PBS. The tissue was incubated for $1 \mathrm{hr}$ at room temperature with avidin-biotinylated horseradish peroxidase (Vectastain ABC kit, Vector Laboratories, Inc., Burlingame, CA, USA), rinsed, incubated with $0.05 \% 3$ 3,3,-diaminobenzidine tetrahydrochloride (DAB substrate kit; BD Pharmingen, San Diego, CA, USA), and then rinsed in PBS to stop the reaction. Sections were mounted and allowed to dry overnight before cover-slipping. Images of CEA lesions were acquired using a Nikon 90i scope at 10X magnification and stitched using Nikon Elements software. CEA lesion reconstructions were drawn on three Paxinos and Watson (1998) plates: $-1.88,-2.56$, and $3.14 \mathrm{~mm}$, from Bregma, and expressed in percentage form relative to the intact CEA.

Neuronal activity in the amygdala (from sham-operated rats) and the pontine nuclei (from sham and lesion rats) was quantified via immunodetection of Fos + cells. Beginning with a pseudo-random tissue section-from Bregma, approximately $-1.9 \mathrm{~mm}$ for the amygdala and $-6.7 \mathrm{~mm}$ for the pontine nuclei-every sixth or fourth section, respectively, was quenched in $0.3 \% \mathrm{H} 2 \mathrm{O} 2$ and blocked in $3 \%$ normal goat serum for $1 \mathrm{hr}$. Sections were incubated for $48 \mathrm{hr}$ at $4{ }^{\circ} \mathrm{C}$ with a rabbit polyclonal antibody against the c-Fos protein (sc-52; Santa Cruz Biotechnology Inc., Santa Cruz, CA, USA) diluted $1 / 2000$ in $3 \%$ normal goat serum. Sections were then incubated at room temperature for $2 \mathrm{hr}$ with a 1/200 dilution of biotinylated antirabbit secondary antibody (BA-1000; Vector Laboratory, Burlingame, CA, USA). Next, sections were incubated for $1 \mathrm{hr}$ at room temperature with the Vectastain $\mathrm{ABC}$ kit, rinsed, incubated with a DAB substrate kit, then rinsed in PBS to stop the reaction. Sections were mounted on slides and, the next day, cover-slipped. For each subject, images were acquired from the PN and amygdala using a Nikon 90i scope at $10 \mathrm{X}$ magnification and stitched using Nikon Elements software. For each tissue section, regions of interest (ROIs) were drawn around specific nuclei in the PN or amygdala. The PN was broken into the medial pontine nuclei (MPN), which spanned the midline, and the lateral pontine nuclei (LPN), which is distributed between both hemispheres (see Fig. 3c). For the amygdala, ROIs were drawn in each hemisphere around the BLA, CEA, and LA (see Fig. 4b). All cell counts were done in a blind manner by one author and a research assistant. Total cell counts were divided by area to generate the number of Fos + cells per unit area $\left(\mathrm{mm}^{2}\right)$. Finally, the percent difference in mean Fos + cell counts from Session 1 to 4 was calculated for each ROI: ([Session 4 mean cell counts / Session 1 mean cell counts] - 1) * 100.

\section{Data analyses}

Statistical analyses were performed with Windows versions of SYSTAT (v. 13) and SPSS (v. 22). Behavioral and IHC results were analyzed using single-factor, mixed design, and repeated-measures ANOVAs, followed, when appropriate, by Tukey-Kramer post hoc testing. A significant post hoc effect implies $p<.05$.

\section{Results}

\section{Lesion reconstructions}

A total of 45 rats underwent surgery in the present study. Thirteen rats were excluded from analysis due to insufficient CEA damage $(<50 \%$ damage across three Paxinos and Watson plates in one or both hemispheres), resulting in the following sample size per group: Sham 1 session $(n=8)$, Lesion 1 session $(n=8)$, Sham 4 session $(n=8)$, and Lesion 4 session $(n=8)$. Figure 1a illustrates CEA lesions across three plates from Paxinos and Watson (1998): -1.88, -2.56, and $-3.14 \mathrm{~mm}$, with the smallest and largest CEA lesions shown in black and gray, respectively. Mean $( \pm S E)$ CEA damage across both one and four session rats was $66 \pm 2 \%$. Minor damage did occur to surrounding structures in addition to the CEA, including the BLA and LA.

\section{Behavioral analyses}

Freezing and eyeblink CR rates were calculated in sham and CEA lesion rats trained with one or four EBC sessions. In rats trained for one session, a single-factor (Lesion) ANOVA revealed significantly reduced session-wide freezing rates in CEA lesion rats $(30.1 \pm 5.7 \%)$ compared to sham rats $(49.5$ $\pm 4.6 \%), F(1,14)=6.88, p<.05$ (data not shown). Figure $2 \mathrm{a}$ illustrates freezing behavior in the four session rats during the first $60 \mathrm{~s}$ of each training session (baseline) and session-wide averages. Baseline freezing, analyzed with a 2 (Lesion) $\times 4$ (Session) repeated measures ANOVA, yielded significant main effects for Lesion only, $F(1,14)=30.47, p<.0001$. Tukey-Kramer post hoc testing indicates the lesion rats froze significantly less than sham rats. Session-wide freezing rates, based on the same ANOVA, yielded a significant Lesion $\times$ Session interaction only, $F(3,42)=5.71, p<.01$. Follow-up one-way (Lesion) ANOVAs indicate the CEA lesion rats froze significantly less than sham controls during the first training session only.

Delay EBC acquisition was also adversely affected by CEA lesions in the four session but not one session rats. For the latter, a single-factor (Lesion) ANOVA applied to eyeblink CR percentage was not statistically significant $(p=.67)$, as expected, considering both groups had low rates of eyeblink CRs on Session 1 (data not shown). In rats trained for four sessions, the 2 (Lesion) $\times 4$ (Session) repeated measures ANOVA revealed significant main effects for Lesion, $F(1$, $14)=5.35, p<.05$, and Session, $F(3,42)=5.11, p<.01$, 


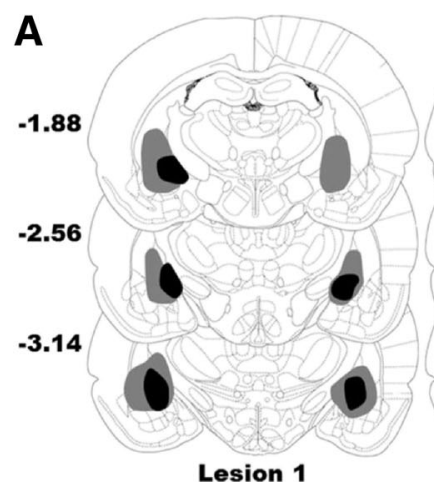

Lesion 1

Fig. 1 Bilateral CEA ibotenic acid lesions. (a) Prior to sacrifice, both sham and lesion rats were submitted to 1 (left) or 4 (right) EBC session(s). Reconstructions are based on three atlas plates (from Bregma: -1.88, 2.56, and $-3.14 \mathrm{~mm}$ ) taken from Paxinos and Watson (1998). The largest lesions are shown in gray and the smallest lesions are shown in black. (b)

but not their interaction. Post hoc testing verified that significantly fewer eyeblink CRs were produced by lesion than sham rats (see Fig. 2b). The same ANOVA applied to CR peak amplitudes, shown in Fig. 2c, found a significant main effect for Session only, $F(3,42)=3.21, p<.05$. Minus a significant
B

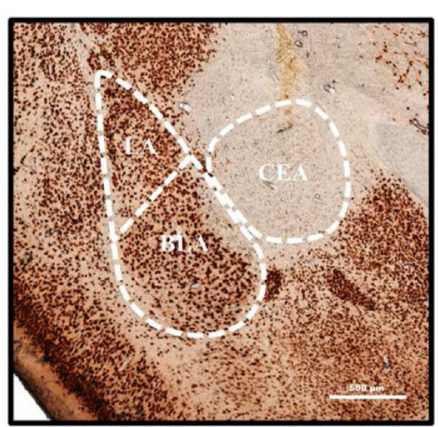

Representative unilateral photomicrograph of a CEA ibotenic acid lesion as indicated by the lack of NeuN-positive neurons. The lateral amygdala (LA), basolateral amygdala (BLA), and central amygdala (CEA) are outlined by dashed lines. Scale bar: $500 \mu \mathrm{m}$

interaction, follow-up one-way (Lesion) ANOVAs on each training session were Bonferroni-corrected, requiring $p<$ .0125 for significance. While CR amplitude was diminished in lesion rats on Session 1, the difference from sham controls was not statistically different $(p=.035)$.
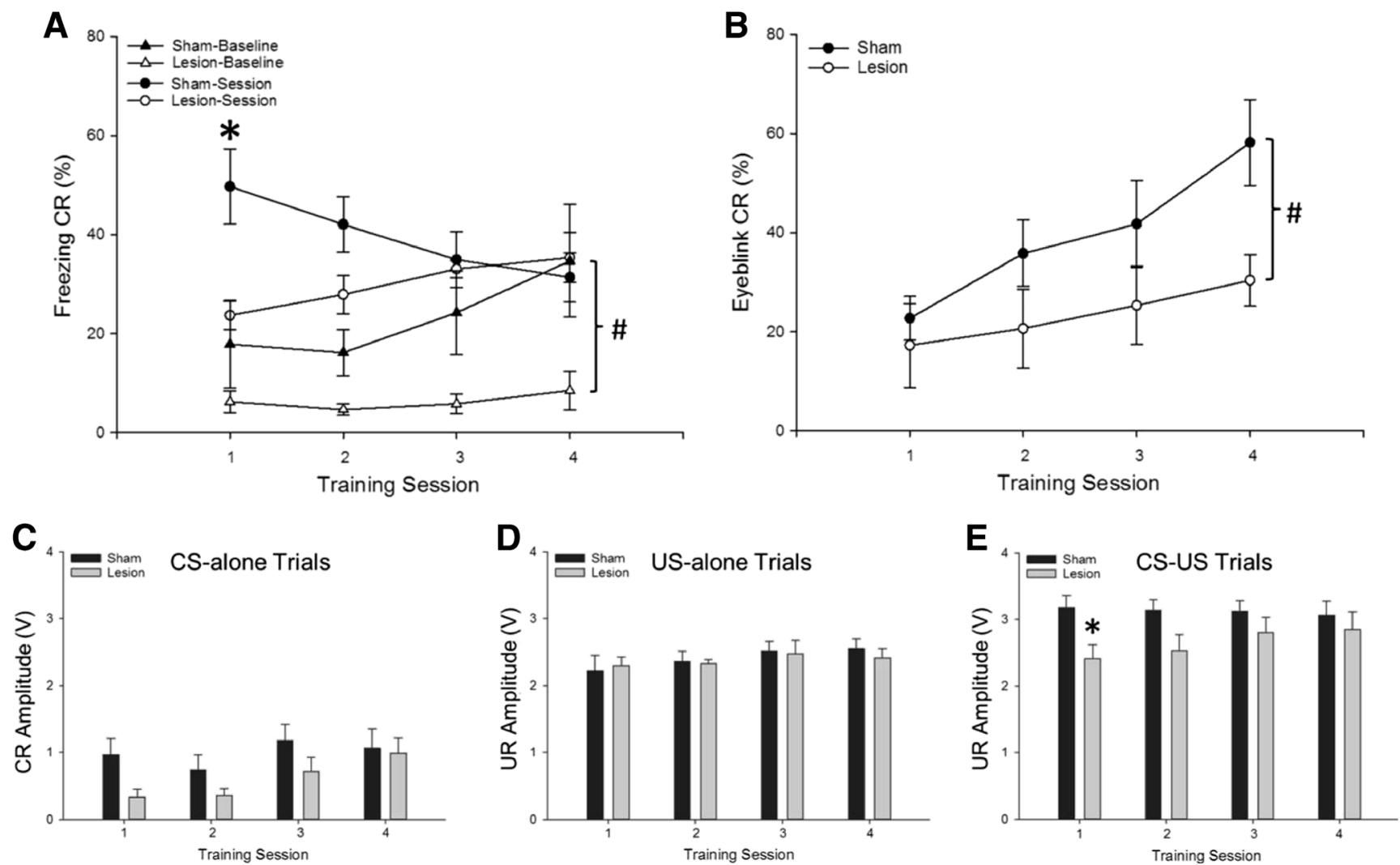

Fig. 2 Freezing behavior and eyeblink conditioned and unconditioned responding in rats trained with $4 \mathrm{EBC}$ sessions. (a) Freezing (mean $\pm S E$ ) was measured during the $60 \mathrm{~s}$ baseline (triangles) or the entire session (circles). Relative to sham controls, results for the CEA lesion rats revealed significantly reduced baseline freezing (pound sign) and, on Session 1, significantly reduced session-wide freezing (asterisk). (b) Eyeblink CR percentages (mean $\pm S E$ ) were significantly reduced in CEA

lesion rats relative to sham controls (pound sign). (c) CR peak amplitude (mean $\pm S E$ ) was not significantly altered in CEA lesion rats. (d) UR peak amplitude (mean $\pm S E$ ), on US-alone trials, was not significantly altered in CEA lesion rats. (e) UR peak amplitude (mean $\pm S E$ ) during CS-US trials was significantly reduced, on Session 1 only, in lesion rats relative to sham controls (asterisk) 
Eyeblink UR peak amplitudes were also analyzed in sham and lesion rats based on US-alone and CS-US paired trials. The former reflects UR strength while the latter measures associative UR facilitation due to the fear-enhancing effects of the tone CS following its pairing with the aversive US (Brown, Kalish, \& Farber, 1951; Choi, Lindquist, \& Brown, 2001). Results for US-alone trials, based on 2 (Lesion) $\times 4$ (session) repeated measures ANOVAs, uncovered a significant main effect for Session only, $F(3,42)=3.32, p<.05$, signifying CEA lesions had no deleterious effect on processing or responding to the US signal (see Fig. 2d). There was, however, a significant Lesion $\times$ Session interaction when UR amplitude was measured following presentation of the tone CS, $F(3,42)=2.92, p<.05$. Follow-up one-way ANOVAs indicate associative UR facilitation was significantly reduced in lesion rats on Session 1 only (see Fig. 2e). Together, the data suggest that CEA lesions had no effect on UR production but did diminish the ability of the CS to potentiate the eyeblink UR.

\section{Immunohistochemical analyses}

PN cell counts were completed by one author and a research assistant blind to each rat's experimental group. The interrater coefficient of determination, based on a subset of ROIs, was $r^{2}$ $=0.93$. There has been a debate in the literature regarding whether acoustic reactivity is restricted to the LPN or distributed throughout the entire PN-thus, Fos+ cells in the LPN and MPN were initially counted and analyzed independently (see Fig. 3c). ROI area encompassed by the LPN (summed across both hemispheres) and MPN was compared first, using a 2 (Lesion) $\times 2($ PN region $) \times 2$ (Session) multifactorial ANOVA. Results revealed a significant main effect for PN region only, $F(1,166)=24.34, p<.0001$, with LPN area $(0.90 \pm .03 \mathrm{~mm})$ significantly smaller than MPN area $(1.10$ $\pm .03 \mathrm{~mm}$; data not shown). Fos + cell counts normalized to LPN and MPN area were analyzed next using the same multifactor ANOVA. Results revealed significant main effects for PN region, $F(1,166)=5.87, p<.05$ and Lesion, $F(1,166)=$ $42.59, p<.001$, but no interaction. Post hoc testing verified the MPN contains significantly fewer Fos + cells than the LPN (see Fig. 3a, left) and, across both regions, the CEA lesion rats have significantly fewer Fos + cells than sham rats (see Fig. 3a, right). Cell counts were next examined for the entire PN in subjects submitted to one or four EBC sessions. Single-factor (Lesion) ANOVAs indicate the total number of Fos + cells was significantly reduced in CEA lesion rats for Session 1, $F(1$, $40)=12.18, p<.001$, and Session 4, $F(1,39)=14.34, p<$ .001 (see Fig. 3b). Reduced auditory-evoked PN neuronal reactivity is proposed to contribute to the retardation in $\mathrm{EBC}$ acquisition seen in lesion rats (see Fig. 2b). Percent difference scores-based on between-subject differences in mean PN Fos + cell counts - indicate cell counts were decreased from
Session 1 to 4 by just $2.4 \%$ in sham rats and increased by $2.2 \%$ in lesion rats.

Finally, the number of amygdala Fos + cells following Sessions 1 and 4 in sham rats was quantified in three amygdala nuclei: the BLA, CEA, and LA. Single-factor (Session) ANOVAs indicate there were significant (between-subject) decreases from Session 1 to 4 in the BLA, $F(1,124)=4.58$, $p<.05$, CEA, $F(1,124)=13.89, p<.001$, and LA, $F(1,124)$ $=10.69, p<.001$ (see Fig. 4a). The percent difference scores indicate Fos + cell counts were decreased from Session 1 to 4 by $18.2 \%$ in the BLA, $40.0 \%$ in the CEA, and $35.7 \%$ in the LA. Results confirm amygdala activation was diminished in a nucleus-specific manner across the four EBC sessions.

\section{Discussion}

Current results indicate that elevated fear responding preceded the emergence of eyeblink CRs in sham control rats, consistent with two-stage models of aversive Pavlovian conditioning (Boele et al., 2010; Lennartz \& Weinberger, 1992; Mintz \& Wang-Ninio, 2001; Rescorla \& Solomon, 1967; Thompson et al., 1987; Wagner \& Brandon, 1989). Bilateral neurotoxic CEA lesions were assessed for their effects on conditioned fear (freezing), CS-mediated UR facilitation, PN acoustic reactivity, and delay EBC acquisition. The lesions impeded feardependent behavior and retarded the emergence of eyeblink CRs across four EBC sessions (see Fig. 2). CEA lesions also significantly reduced the number of Fos $+\mathrm{PN}$ cells, suggesting the amygdala may normally enhance CS saliency and facilitate EBC acquisition by increasing the population of tonereactive PN neurons (see Fig. 3). Finally, bigger decreases in Fos + cell counts in the sham rats were seen following Session 4 in LA and CEA than the BLA, compared to Session 1 (see Fig. 4). Taken together, we propose delay EBC proceeds through two well-documented stages, followed by a third putative stage involving cerebellar-mediated emotional regulation.

Two-stage models of aversive Pavlovian conditioning conceptualize sensorimotor learning to pass through two sequential stages (Neufeld \& Mintz, 2001; Thompson et al., 1987; Wagner \& Brandon, 1989). Broadly speaking, in response to the aversive US the amygdala is proposed to rapidly initiate a state of "conditioned arousal" early in EBC (Kapp, Wilson, Pascoe, Supple, \& Whalen, 1990; Thompson et al., 1987), enhancing the salience of relevant stimuli and the rate at which eyeblink CRs emerge. Many studies have documented rapid changes in both autonomic and defensive behavior early in EBC (e.g., Lee \& Kim, 2004; Prokasy, 1972), mediated by the CEA and its efferent projections to a variety of hypothalamic and brainstem targets (Applegate, Frysinger, Kapp, \& Gallagher, 1982; Fox, Oler, Tromp, Fudge, \& Kalin, 2015). What exactly drives the rapid increase in fear responding is 

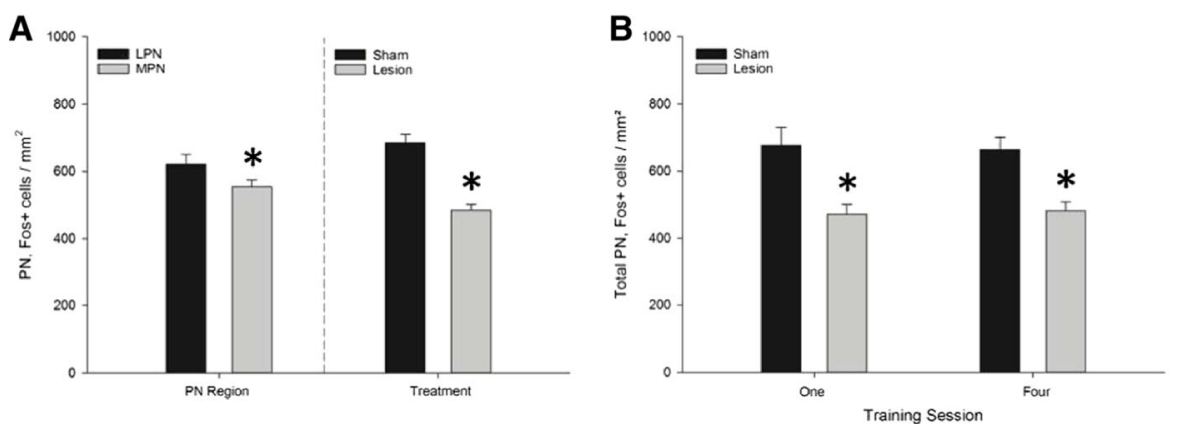

C

Fig. 3 Bilateral PN Fos + cell counts in sham and lesion rats sacrificed after one or four EBC session(s). (a) Left side: There were significantly fewer MPN Fos + cells (mean $\pm S E$ ) compared to counts from the LPN. Right side: Fos + cell counts (mean $\pm S E$ ) from both LPN and MPN were significantly reduced in CEA lesion rats. (b) Fos + cell counts (mean $\pm S E$ )

not entirely clear, however - it likely reflects a combination of unconditioned (or generalized) fear and conditioned fear specific to the tone CS and/or training context. Britton and Astheimer (2004) explored this issue by measuring CSevoked freezing during a $12 \mathrm{~s}$ tone $\mathrm{CS}$ inserted into each EBC session or, separately, in a novel context. Conditioned freezing in the training and novel contexts was consistently higher in adult rats trained with CS-US paired trials than CSUS unpaired or US-alone trials. Further the decrease in freezing behavior seen over subsequent training sessions was dependent on the CS-US contingency - that is, rats trained with unpaired or US-alone trials demonstrated lower but more sustained freezing levels. In other words, the gradual decline in conditioned fear seen over training may be learning dependent and not the result of simple habituation. Inasmuch, we propose the initial elevation and subsequent decrease in session-wide freezing rates seen in sham rats is due, at least in part, to the associative nature of the CS-US relationship. from the entire PN were significantly reduced in lesion rats following session one and four. For all figures, an asterisk indicates a significant effect. (c) Representative photomicrograph of Fos + cells in the LPN and MPN (outlined by dashed lines) in one hemisphere of a sham rat. Scale bar: $500 \mu \mathrm{m}$

In line with previous research (Blankenship et al., 2005; Lee \& Kim, 2004; Weisz et al., 1992) CEA lesions significantly retarded the emergence of eyeblink CRs (see Fig. 2b). Moreover, once a conditioned blink was generated, there was a trend toward smaller CR peak amplitudes in the lesion rats (Fig. 2c). No experimental group differences were seen following US-alone trials, however, suggesting US processing and UR production is unimpeded by CEA lesions (see Fig. 2d). As for conditioned fear, session-wide freezing rates were significantly reduced, relative to sham controls, during the first training session only (see Fig. 2a). The CEA is also capable of facilitating a variety of URs or reflexes (e.g., whole body acoustic startle) when elicited in the presence of a feareliciting CS (Choi et al., 2001; Hitchcock \& Davis, 1991). In eyeblink conditioned rabbits, the UR was reportedly enhanced within the first 5 to 12 trials, with larger amplitudes on CS-US paired than US-alone trials (Weisz \& McInerney, 1990). As illustrated in Fig. 2e, CEA lesion rats produced little
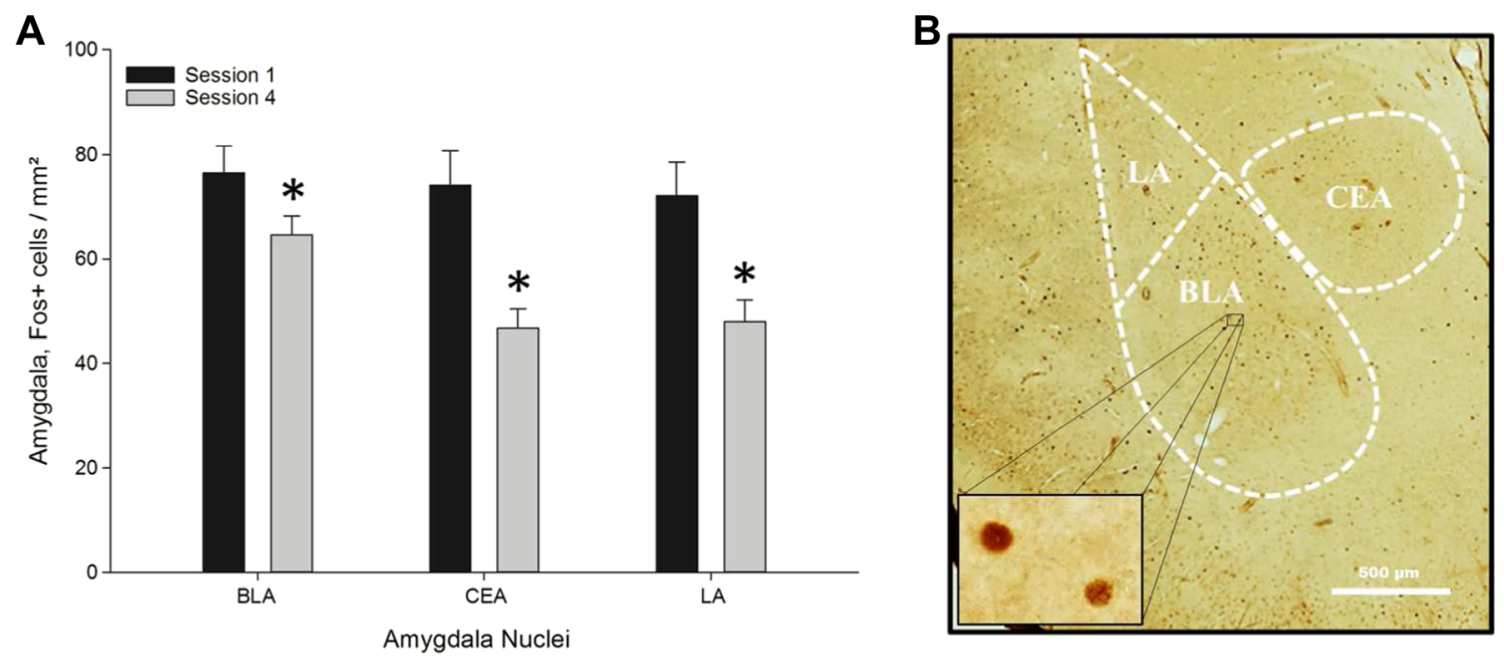

Fig. 4 Bilateral amygdala Fos + cell counts in sham lesion rats sacrificed after one or four EBC session(s). (a) Fos + cell counts (mean $\pm S E$ ) were significantly decreased from Session 1 to 4 in the basolateral amygdala (BLA, left), the central amygdala (CEA; middle), and the lateral

amygdala (LA; right), as indicated by asterisks. (b) Representative unilateral photomicrograph of Fos + cells in the LA, BLA, and CEA (outlined by dashed lines) of a sham rat. Inset shows Fos+ BLA cells at high (60X) magnification. Scale bar: $500 \mu \mathrm{m}$ 
associative UR facilitation on the first training session. The increase in UR amplitude provided by the CS (i.e., relative to US-alone trials) was negligible $(5.0 \%)$ compared to the increase seen in sham rats $(42.7 \%)$. This result runs counter to two prior EBC studies, which found no reliable experimental group differences in UR amplitude (based on CS-US trials), when the CEA was pharmacologically inactivated in young rats or, in adult rats, the whole amygdala was damaged (Lee \& Kim, 2004; Ng \& Freeman, 2014). Nevertheless, current data indicate that the loss of potentiated freezing and reflexive responding observed in lesion rats was, in both cases, most pronounced on the first training session.

The acoustic CS signal is relayed through the medial thalamic nuclei, the cochlear nucleus, the nucleus of the lateral lemniscus, and finally to the PN, the last precerebellar nucleus that conveys acoustic signals to the cerebellum (Freeman \& Steinmetz, 2011). Auditory cues were previously reported to be selectively relayed to the cerebellum through the LPN (Bao, Chen, \& Thompson, 2000; Steinmetz et al., 1987), while other research found auditory-evoked responses throughout both the LPN and MPN (Cartford, Gohl, Singson, \& Lavond, 1997). Based on multi-unit recordings, Taub and Mintz (2010) argued that amygdala-dependent CRs enhance tone-mediated reactivity in PN neurons along most of its medial-lateral axis. As seen in Fig. 3, CEA lesion rats likewise had a significant reduction in Fos + neurons in both the LPN and MPN. Interestingly, the mean area of the LPN was significantly smaller than the MPN, yet it also had significantly more Fos + cells, suggesting the LPN may contain a higher concentration of acoustic-reactive neurons than the MPN.

The PN is thought to be innervated and diffusely influenced by the CEA through both monosynaptic and polysynaptic projections. As an example, during fear conditioning the CEA could stimulate acetylcholine release from the nucleus basalis, biasing the firing of auditory cortex neurons - which innervate the entire PN (Perales, Winer, \& Prieto, 2006; Wiesendanger \& Wiesendanger, 1982) - toward the frequency of the tone CS (Weinberger, 1998, 2004). CEA lesions reduced the number of $\mathrm{PN}$ Fos + cells and decelerated EBC acquisition, as predicted. How PN neuronal kinetics were altered by CEA lesions is not known - that is, the reduction in PN c-Fos immunostaining could be due to diminished toneevoked reactivity or could reflect a more low-level effect such as a reduction in basal neuronal activity. Regardless of the precise mechanism, however, CEA lesions are proposed to diminish the neuronal saliency of the CS signal, impeding cerebellar CS-US associative plasticity.

We hypothesized PN acoustic reactivity was amplified via CEA projection neurons, concomitant with autonomic and behavioral fear CR expression. From Session 1 to 4 in sham rats, the number of Fos + cells in the CEA fell by $40.0 \%$ while PN cell counts decreased by only $2.4 \%$, a result that is hard to reconcile if the CEA is responsible for increasing the population of tone reactive cells. IP-dependent changes in spiking activity, tied to eyeblink CR production, have been recorded in numerous loci throughout the brain, including for instance, hippocampal CA1 neurons (reviewed in Lavond \& Cartford, 2000). The proportion of $P N$ neurons that discharge each time an eyeblink CR is generated, modeling its amplitude and time course, also increases over training (Bao et al., 2000). While speculative, perhaps CEA amplification of tone-evoked PN reactivity is diminished over training, but the loss is supplemented each time a conditioned blink is emitted by IPgenerated excitatory feedback.

Rats exhibit conditioned fear to the training context early in EBC in that they show elevated freezing before and after CSUS presentations (Britton \& Astheimer, 2004; Ng \& Freeman, 2014). We propose that the context-US memory grows in strength over training, such that subsequent exposures to the context - which includes both the physical chamber and the handling required to attach each subject's headstage — leads to faster retrieval and higher baseline freezing rates (see Fig. 2a). Context-dependent fear is known to influence the emission of eyeblink CRs - for example, switching eyeblink conditioned rats or rabbits to a novel context leads to a decrease in the number of emitted eyeblink CRs (Lindquist, 2013; Penick \& Solomon, 1991). The fact that baseline freezing levels increased over training sessions in sham and lesion rats suggests the amygdala may facilitate the context-US association at a slower rate than the CS-US association. Indeed, the hippocampus has been proposed to process contextual information in competition with the cerebellar CS-US association (Lee \& Kim, 2004), such that hippocampal lesions actually facilitate eyeblink CR acquisition during delay EBC (Christiansen and Schmajuk 1992; Port, Mikhail, \& Patterson, 1985; Schmaltz \& Theios, 1972).

Anatomically, the amygdala and hippocampus share extensive connections (Pitkanen, 2000; Pitkanen, Pikkarainen, Nurminen, \& Ylinen, 2000). All major amygdala nuclei receive input from the hippocampus, though electrophysiological recordings suggest the BLA innervates and influences hippocampal neuronal activity to a greater degree than LA or CEA (Blankenship et al., 2005; Ikegaya, Saito, \& Abe, 1994; Racine, Milgram, \& Hafner, 1983). In rats sacrificed following Session 1 and 4, the BLA showed the smallest decrease in Fos + cell counts (18.2\%), about half that seen for the CEA (40.0 \%) and LA (35.7\%). Considering the BLA is thought to acquire and store information about the emotionally arousing properties of specific contexts (Huff \& Rudy, 2004; Zelikowsky, Hersman, Chawla, Barnes, \& Fanselow, 2014), the BLA could conceivably interact with the hippocampus throughout EBC, modulating and/or storing the context-US memory (Akirav \& Richter-Levin, 1999; Kochli, Thompson, Fricke, Postle, \& Quinn, 2015). Inconsistent with this idea, however, is the impaired baseline freezing seen in 
CEA lesion rats (whose BLA was mostly intact) across training, relative to the sham controls (see Fig. 2a).

Altogether, results from the current study suggest the amygdala and its diffuse CEA output projections are capable of facilitating cerebellar sensorimotor learning via the enhancement of CS-mediated brainstem activity. Intriguingly, a recent study found that experimental stimulation of IP projection neurons (mimicking an eyeblink CR) can inhibit CEA neuronal responding to an aversive periorbital US, possibly as a means to regulate fear responding as conditioned eyeblinks grow in number (Magal \& Mintz, 2014). It raises the interesting possibility that the robust reduction in Fos+ cells seen in the CEA (and possibly LA) from Session 1 to 4 - and the concomitant decrease in conditioned freezing - is the result of learning-dependent negative feedback from the cerebellum, representing a third stage of aversive Pavlovian conditioning. As first proposed by Mintz and colleagues (Erez, Gordon, Sever, Sadeh, \& Mintz, 2004; Mintz \& Wang-Ninio, 2001), we suggest the two stage model should be reconceptualized as a three-stage model: rapid emotional responding in stage one, which enhances discrete motor learning in stage two, which diminishes emotional reactivity in stage three. Dynamic and reciprocal amygdalar-cerebellar modulation of this type would provide the immediate benefits of conditioned fear or arousal, while also ensuring the heightened emotional state is ultimately stabilized and returned to baseline levels. Absent such a mechanism, following cerebellar damage for instance (Lavond, Lincoln, McCormick, \& Thompson, 1984), chronic increases in amygdala activation and emotional arousal could prime or even provoke various affective/anxiety disorders (Kim et al., 2011; Magal \& Mintz, 2014).

\begin{abstract}
Acknowledgments The authors thank Roxanne Kaercher and Jennifer Coppola for assistance running rats, Jasminder Virk and Benjamin Johnston for assistance with lesion reconstructions, and Michael (Alex) Lee for assistance with pontine nuclei cell counts. No authors have a conflict of interest for any research in the current manuscript, including any financial, personal or other relationships with other people or organizations.
\end{abstract}

\section{References}

Aitkin, L. M., \& Boyd, J. (1978). Acoustic input to the lateral pontine nuclei. Hearing Research, 1, 67-77.

Akirav, I., \& Richter-Levin, G. (1999). Biphasic modulation of hippocampal plasticity by behavioral stress and basolateral amygdala stimulation in the rat. The Journal of Neuroscience, 19, 1053010535.

Applegate, C. D., Frysinger, R. C., Kapp, B. S., \& Gallagher, M. (1982). Multiple unit activity recorded from amygdala central nucleus during Pavlovian heart rate conditioning in rabbit. Brain Research, 238, $457-462$.
Bao, S., Chen, L., \& Thompson, R. F. (2000). Learning- and cerebellumdependent neuronal activity in the lateral pontine nucleus. Behavioral Neuroscience, 114, 254-261.

Berger, T. W., Alger, B., \& Thompson, R. F. (1976). Neuronal substrate of classical conditioning in the hippocampus. Science, 192, 483-485.

Berthier, N. E., \& Moore, J. W. (1990). Activity of deep cerebellar nuclear cells during classical conditioning of nictitating membrane extension in rabbits. Experimental Brain Research, 83, 44-54.

Blair, H. T., Sotres-Bayon, F., Moita, M. A., \& LeDoux, J. E. (2005). The lateral amygdala processes the value of conditioned and unconditioned aversive stimuli. Neuroscience, 133, 561-569.

Blankenship, M. R., Huckfeldt, R., Steinmetz, J. J., \& Steinmetz, J. E. (2005). The effects of amygdala lesions on hippocampal activity and classical eyeblink conditioning in rats. Brain Research, 1035, 120 130.

Boele, H. J., Koekkoek, S. K., \& De Zeeuw, C. I. (2010). Cerebellar and extracerebellar involvement in mouse eyeblink conditioning: The ACDC model. Frontiers in Cellular Neuroscience, 3, 1-13.

Britton, G. B., \& Astheimer, L. B. (2004). Fear develops to the conditioned stimulus and to the context during classical eyeblink conditioning in rats. Integrative Physiological \& Behavioral Science, 39, 295-306.

Brown, J. S., Kalish, H. I., \& Farber, I. E. (1951). Conditioned fear as revealed by magnitude of startle response to an auditory stimulus. Journal of Experimental Psychology, 41, 317-328.

Bullitt, E. (1990). Expression of c-fos-like protein as a marker for neuronal activity following noxious stimulation in the rat. The Journal of Comparative Neurology, 296, 517-530.

Cartford, M. C., Gohl, E. B., Singson, M., \& Lavond, D. G. (1997). The effects of reversible inactivation of the red nucleus on learningrelated and auditory-evoked unit activity in the pontine nuclei of classically conditioned rabbits. Learning \& Memory, 3, 519-531.

Chachich, M., \& Powell, D. A. (1998). Both medial prefrontal and amygdala central nucleus lesions abolish heart rate classical conditioning, but only prefrontal lesions impair reversal of eyeblink differential conditioning. Neuroscience Letters, 257, 151-154.

Choi, J. S., Lindquist, D. H., \& Brown, T. H. (2001). Amygdala lesions prevent conditioned enhancement of the rat eyeblink reflex. Behavioral Neuroscience, 115, 764-775.

Christian, K. M., \& Thompson, R. F. (2003). Neural substrates of eyeblink conditioning: Acquisition and retention. Learning \& Memory, 11, 427-455.

Christiansen, B. A., \& Schmajuk, N. A. (1992). Hippocampectomy disrupts the topography of the rat eyeblink response during acquisition and extinction of classical conditioning. Brain Research, 595, 206 214.

Duvarci, S., \& Pare, D. (2014). Amygdala microciruits controlling learned fear. Neuron, 82, 966-980.

Erez, O., Gordon, C. R., Sever, J., Sadeh, A., \& Mintz, M. (2004). Balance dysfunction in childhood anxiety: Findings and theoretical approach. Journal of Anxiety Disorders, 18, 341-356.

Fanselow, M. S. (1980). Conditional and unconditional components of post-shock freezing. Pavlovian Journal of Biological Science, 15(4), $177-182$

Fanselow, M. S., \& Poulos, A. M. (2005). The neuroscience of mammalian associative learning. Annual Review of Psychology, 56, $207-$ 234.

Fox, A. S., Oler, J. A., Tromp, D. P., Fudge, J. L., \& Kalin, N. H. (2015). Extending the amygdala in theories of threat processing. Trends in Neurosciences, 38, 319-329.

Freeman, J. H., \& Steinmetz, A. B. (2011). Neural circuitry and plasticity mechanisms underlying delay eyeblink conditioning. Learning \& Memory, 18, 666-677.

Green, J. T., \& Steinmetz, J. E. (2005). Purkinje cell activity in the cerebellar anterior lobe after rabbit eyeblink conditioning. Learning \& Memory, 12, 260-269. 
Hitchcock, J. M., \& Davis, M. (1991). Efferent pathway of the amygdala involved in conditioned fear as measured with the fear-potentiated startle paradigm. Behavioral Neuroscience, 105, 826-842.

Huff, N. C., \& Rudy, J. W. (2004). The amygdala modulates hippocampus-dependent context memory formation and stores cue-shock associations. Behavioral Neuroscience, 118, 53-62.

Ikegaya, Y., Saito, H., \& Abe, K. (1994). Attenuated hippocampal longterm potentiation in basolateral amygdala-lesioned rats. Brain Research, 656, 157-164.

Kandler, K., \& Herbert, H. (1991). Auditory projections from the cochlear nucleus to pontine and mesencephalic reticular nuclei in the rat. Brain Research, 562, 230-242.

Kapp, B. S., Wilson, A., Pascoe, J. P., Supple, W. F., \& Whalen, P. J. (1990). Neuroanatomical systems analysis of conditioned bradycardia in the rabbit. In M. Gabriel \& J. W. Moore (Eds.), Learning and computational neuroscience: Foundations of adaptive networks (pp. 53-90). Cambridge, MA: MIT Press.

Kim, M. J., Loucks, R. A., Palmer, A. L., Brown, A. C., Solomon, K. M., Marchante, A. N., \& Whalen, P. J. (2011). The structural and functional connectivity of the amygdala: From normal emotion to pathological anxiety. Behavioral Brain Research, 223, 403-410.

Kochli, D. E., Thompson, E. C., Fricke, E. A., Postle, A. F., \& Quinn, J. J. (2015). The amygdala is critical for trace, delay, and contextual fear conditioning. Learning \& Memory, 22, 92-100.

Koo, J.-W., Han, J.-S., \& Kim, J. J. (2004). Selective neurotoxic lesions of basolateral and central nuclei of the amygdala produce differential effects on fear conditioning. The Journal of Neuroscience, 24, 7654-7662.

Lavond, D. G., \& Cartford, M. C. (2000). Eye blink circuitry: Training, lesion, and reversible lesion experiments. In D. S. Woodruff-Pak \& J. E. Steinmetz (Eds.), Eyeblink classical conditioning: Animals (pp. 51-80). Norwell, MA: Kluwer Academic Publishers.

Lavond, D. G., Lincoln, J. S., McCormick, D. A., \& Thompson, R. F. (1984). Effect of bilateral lesions of the dentate and interpositus cerebellar nuclei on conditioning of heart-rate and nictitating membrane/eyelid resonses in the rabbit. Brain Research, 305, $323-330$.

LeDoux, J. E. (2014). Coming to terms with fear. Proceedings of the National Academy of Science of the United States of America, 111, 2871-2878

Lee, T., \& Kim, J. J. (2004). Differential effects of cerebellar, amygdalar, and hippocampal lesions on classical eyeblink conditioning in rats. The Journal of Neuroscience, 24, 3242-3250.

Lennartz, R. C., \& Weinberger, N. M. (1992). Analysis of response systems in Pavlovian conditioning reveals rapidly versus slowly acquired conditioned responses: Support for two factors, implications for behavior and neurobiology. Psychobiology, 20, 93-119.

Lindquist, D. H. (2013). Hippocampal-dependent Pavlovian conditioning in adult rats exposed to binge-like doses of ethanol as neonates. Behavioral Brain Research, 242, 191-199.

Lindquist, D. H., Mahoney, L. P., \& Steinmetz, J. E. (2010). Conditioned fear in adult rats is facilitated by the prior acquisition of a classically conditioned motor response. Neurobiology of Learning and Memory, 94, 167-175.

Magal, A., \& Mintz, M. (2014). Inhibition of the amygdala central nucleus by stimulation of cerebellar output in rats: A putative mechanism for extinction of the conditioned fear response. European Journal of Neuroscience, 40, 3548-3555.

McCormick, D. A., \& Thompson, R. F. (1984). Neuronal responses of the rabbit cerebellum during acquisition and performance of a classically conditioned nictitating membrane-eyelid response. The Journal of Neuroscience, 4, 2811-2822.

Mihailoff, G. A., Kosinski, R. J., Azizi, S. A., \& Border, B. G. (1989). Survey of noncortical afferent projections to the basilar pontine nuclei: A retrograde tracing study in the rat. The Journal of Comparative Neurology, 282, 617-643.
Mintz, M., \& Wang-Ninio, Y. (2001). Two-stage theory of conditioning: Involvement of the cerebellum and the amygdala. Brain Research, 897, 150-156.

Mullen, R. J., Buck, C. R., \& Smith, A. M. (1992). NeuN, a neuronal specific nuclear protein in vertebrates. Development, 116, 201-211.

Neufeld, M., \& Mintz, M. (2001). Involvement of the amygdala in classical conditioning of eyeblink response in the rat. Brain Research, 889, 112-117.

Ng, K. H., \& Freeman, J. H. (2014). Amygdala inactivation impairs eyeblink conditioning in developing rats. Developmental Psychobiology, 56, 999-1007.

Paxinos, G., \& Watson, C. (1998). The rat brain: In stereotaxic coordinates. Salt Lake City, UT: Academic Press.

Penick, S., \& Solomon, P. R. (1991). Hippocampus, context, and conditioning. Behavioral Neuroscience, 105, 611-617.

Penzo, M. A., Robert, V., \& Li, B. (2014). Fear conditioning potentiates synaptic transmission onto long-range projection neurons in the lateral subdivision of central amygdala. The Journal of Neuroscience, 34, 2432-2437.

Perales, M., Winer, J. A., \& Prieto, J. J. (2006). Focal projections of cat auditory cortex to the pontine nuclei. The Journal of Comparative Neurology, 497, 959-980.

Perrett, S. P., Ruiz, B. P., \& Mauk, M. D. (1993). Cerebellar cortex lesions disrupt learning-dependent timing of conditioned eyelid responses. The Journal of Neuroscience, 13, 1708-1718.

Pitkanen, A. (2000). Connectivity of the rat amygdaloid complex. In J. P. Aggleton (Ed.), The amygdala: A functional analysis (pp. 31-116). New York, NY: Oxford University Press.

Pitkanen, A., Pikkarainen, M., Nurminen, N., \& Ylinen, A. (2000). Reciprocal connections between the amygdala and hippocampal formation, perirhinal cortex, and postrhinal cortex in rat. Annals of the New York Academy of Sciences, 911, 369-391.

Port, R. L., Mikhail, A. A., \& Patterson, M. M. (1985). Differential effects of hippocampectomy on classically conditioned rabbit nictitating membrane response related to interstimulus interval. Behavioral Neuroscience, 99, 200-208.

Prokasy, W. F. (1972). Developments with the two-phase model applied to human eyelid conditioning. In A. H. Black \& W. F. Prokasy (Eds.), Classical conditioning II: Current research and theory (pp. 199-247). New York, NY: Appleton-Century-Crofts.

Racine, R. J., Milgram, N. W., \& Hafner, S. (1983). Long-term potentiation phenomena in the rat limbic forebrain. Brain Research, 260, 217-231

Rescorla, R. A., \& Solomon, R. L. (1967). Two-process learning theory: Relationships between Pavlovian conditioning and instrumental learning. Psychological Review, 74, 151-182.

Rorick-Kehn, L. M., \& Steinmetz, J. E. (2005). Amygdalar unit activity during three learning tasks: Eyeblink classical conditioning, Pavlovian fear conditioning, and signaled avoidance conditioning. Behavioral Neuroscience, 119, 1254-1276.

Rudy, J. W. (2009). Context representations, context functions, and the parahippocampal-hippocampal system. Learning \& Memory, 16, 573-585.

Sah, P., \& Armentia, M. L. D. (2003). Excitatory synaptic transmission in the lateral and central amygdala. Annals of the New York Academy of Sciences, 985, 67-77.

Schmaltz, L. W., \& Theios, J. (1972). Acquisition and extinction of a classically conditioned response in hippocampectomized rabbits (Oryctolagus cuniculus). Journal of Comparative \& Physiological Psychology, 79, 328-333.

Schneiderman, N. (1972). Response system divergencies in aversive classical conditioning. In A. H. Black \& W. F. Prokasy (Eds.), Classical conditioning II: Current research and theory (pp. 341-376). New York, NY: Appleton-Century-Crofts.

Stanton, M. E. (2000). Multiple memory systems, development and conditioning. Behavioral Brain Research, 110, 25-37. 
Steinmetz, J. E., \& Lindquist, D. H. (2009). Neuronal basis of learning. In G. G. Berntson \& J. T. Cacioppo (Eds.), Handbook of neuroscience for the behavioral sciences (pp. 507-527). Hoboken, NJ: John Wiley \& Sons.

Steinmetz, J. E., Logan, C. G., Rosen, D. J., Thompson, J. K., Lavond, D. G., \& Thompson, R. F. (1987). Initial localization of the acoustic conditioned stimulus projection system to the cerebellum essential for classical eyelid conditioning. Proceedings of the National Academy of Sciences, 84, 3531-3535.

Steinmetz, J. E., \& Sengelaub, D. R. (1992). Possible conditioned stimulus pathway for classical eyelid conditioning in rabbits. I. anatomical evidence for direct projections from the pontine nuclei to the cerebellar interpositus nucleus. Behavioral and Neural Biology, 57, 103-115.

Taub, A. H., \& Mintz, M. (2010). Amygdala conditioning modulates sensory input to the cerebellum. Neurobiology of Learning and Memory, 94, 521-529.

Thompson, R. F., Donegan, N. H., Clark, G. A., Lavond, D. G., Lincoln, J. S., Madden, J. IV, . . McCormick, D. A. (1987). Neuronal substrates of discrete, defensive conditioned reflexes, conditioned fear states, and their interactions in the rabbit. In I. Gormezano, W. F. Prokasy, \& R. F. Thompson (Eds.), Classical conditioning (pp. 371399). Hillsdale, NJ: Erlbaum.

Thompson, R. F., \& Steinmetz, J. E. (2009). The role of the cerebellum in classical conditioning of discrete behavioral responses. Neuroscience, 162, 732-755.

Türker, K. S., \& Miles, T. S. (1986). Climbing fiber lesions disrupt conditioning of the nictating membrane response in the rabbit. Brain Research, 363, 376-378.
Wagner, A. R., \& Brandon, S. E. (1989). Evolution of a structured connectionist model of Pavlovian conditioning (AESOP). In S. B. Klein \& R. R. Mowrer (Eds.), Contemporary learning theories: Pavlovian conditioning and the status of traditional learning theory (pp. 149190). Hillsdale, NJ: Erlbaum.

Weinberger, N. M. (1998). Physiological memory in primary auditory cortex: Characteristics and mechanisms. Neurobiology of Learning and Memory, 70, 226-251.

Weinberger, N. M. (2004). Specific long-term memory traces in primary audtiory cortex. Nature Reviews. Neuroscience, 5, 279-290.

Weisz, D. J., Harden, D. G., \& Xiang, Z. (1992). Effects of amygdala lesions on reflex facilitation and conditioned response acquisition during nictitating membrane response conditioning in rabbit. Behavioral Neuroscience, 106, 262-273.

Weisz, D. J., \& McInerney, J. (1990). An associative process maintains reflex facilitation of the unconditioned nictitating membrane response during the early stages of training. Behavioral Neuroscience, 104, 21-27.

Wiesendanger, R., \& Wiesendanger, M. (1982). The corticopontine system in the rat. II. The projection pattern. Journal of Comparative Neurology, 208, 227-238.

Yeo, C. H., Hardiman, M. J., \& Glickstein, M. (1986). Classical conditioning of the nictitating membrane response of the rabbit. IV. lesions of the inferior olive. Experimental Brain Research, 63, 81-92.

Zelikowsky, M., Hersman, S., Chawla, M. K., Barnes, C. A., \& Fanselow, M. S. (2014). Neuronal ensembles in amygdala, hippocampus, and prefrontal cortex track differential components of contextual fear. The Journal of Neuroscience, 34, 8462-8466. 